\title{
A remark on our paper "Negative holomorphic curvature and positive canonical bundle"
}

\author{
DAmin Wu AND SHING-Tung YAU
}

\begin{abstract}
This is a continuation of our first paper in [WY16]. There are two purposes of this paper: One is to give a proof of the main result in [WY16] without going through the argument depending on numerical effectiveness. The other one is to provide a proof of our conjecture, mentioned in [TY], where the assumption of negative holomorphic sectional curvature is dropped to quasi-negative. We should note that a solution to our conjecture is also provided by Diverio-Trapani [DT]. Both proofs depend on our argument in [WY16]. But our argument here makes use of the argument given by the second author and Cheng in [CY75].

The proof of Theorem 1 below is obtained by us in 2015, and has been distributed in the community; e.g. it was presented by the second author in the birthday conference of Richard Schoen on June 21, 2015. We have also settled the complete noncompact case, which, together with applications, will appear soon.
\end{abstract}

\section{Negative holomorphic sectional curvature}

By using the argument in [WY16], we can directly derive the following result in the Kähler setting, without using the notion of nefness.

Theorem 1. Let $(X, \omega)$ be a compact Kähler manifold with negative holomorphic sectional curvature. Then $X$ admits a Kähler-Einstein metric of negative Ricci curvature. In particular, the canonical bundle of $X$ is ample.

The first author was partially supported by the NSF grants DMS-1308837 and DMS-1611745. The second author was partially supported by the NSF grant DMS0804454 and DMS-1308244. 
Proof. For $t>0$, consider the Monge-Ampère type equation

$$
\left(t \omega+d d^{c} \log \omega^{n}+d d^{c} u_{t}\right)^{n}=e^{u_{t}} \omega^{n} .
$$

Since $\omega>0$ and $X$ is compact, there exists a sufficiently large constant $t_{1}>1$ such that $t_{1} \omega+d d^{c} \log \omega^{n}>0$ on $X$. Fix an nonnegative integer $k$ and $0<\alpha<1$. Denote by $C^{k, \alpha}(X)$ the Hölder space with respect to fixed metric $\omega$. Define

(1) $I=\left\{t \in\left[0, t_{1}\right] \mid\right.$ there is a $u_{t} \in C^{k+2, \alpha}(X)$ satisfying $(\mathrm{MA})_{t}$ and $\left.t \omega+d d^{c} \log \omega^{n}+d d^{c} u_{t}>0\right\}$.

First, note that $I \neq \emptyset$, since $t_{1} \in I$. Indeed, $(\mathrm{MA})_{t_{1}}$ can be written as

$$
\left(t_{1} \omega+d d^{c} \log \omega^{n}+d d^{c} u_{t_{1}}\right)^{n}=e^{u_{t_{1}}+f_{1}}\left(t_{1} \omega+d d^{c} \log \omega^{n}\right)^{n},
$$

where

$$
f_{1}=\log \frac{\omega^{n}}{\left(t_{1} \omega+d d^{c} \log \omega^{n}\right)^{n}} \in C^{\infty}(X) .
$$

Applying [Yau78] obtains a solution $u_{t_{1}} \in C^{\infty}(X)$.

That $I$ is open in $\left[0, t_{1}\right]$ follows from the implicit function theorem. Indeed, let $t_{0} \in I$ with corresponding function $u_{t_{0}} \in C^{k+2, \alpha}(X)$. Then, there exists a small neighborhood $J$ of $t_{0}$ in $\left[0, t_{1}\right]$ and a small neighborhood $U$ of $u_{t_{0}}$ in $C^{k+2, \alpha}(X)$ such that

$$
t \omega+d d^{c} \log \omega^{n}+d d^{c} v>0 \text { for all } t \in J \text { and } v \in U
$$

Define a map $\Phi: J \times U \rightarrow C^{k, \alpha}(X)$ by

$$
\Phi(t, v)=\log \frac{\left(t \omega+d d^{c} \log \omega^{n}+d d^{c} v\right)^{n}}{\omega^{n}}-v
$$

Then, the linearization is given by

$$
\Phi_{u_{t_{0}}}\left(t_{0}, u_{t_{0}}\right) h=\left.\frac{d}{d s} \Phi\left(t_{0}, u_{t_{0}}+s h\right)\right|_{s=0}=\left(\Delta_{t_{0}}-1\right) h
$$

which is invertible from $C^{k+2, \alpha}(X)$ to $C^{k, \alpha}(X)$, where $\Delta_{t_{0}}$ stands for the Laplacian of metric $t_{0} \omega+d d^{c} \log \omega^{n}+d d^{c} u_{t_{0}}$. Thus, we can apply the implicit function to obtain the openness of $I$. 
The closedness is contained in the proof of Theorem 7 in [WY16]. More precisely, for $t \in I$ we denote

$$
\omega_{t}=t \omega+d d^{c} \log \omega^{n}+d d^{c} u_{t}, \quad \text { and } \quad S=\frac{n \omega_{t}^{n-1} \wedge \omega}{\omega_{t}^{n}},
$$

Then (MA) $)_{t}$ becomes $\omega_{t}^{n}=e^{u_{t}} \omega^{n}$. It follows that

$$
d d^{c} \log \omega_{t}^{n}=d d^{c} \log \omega^{n}+d d^{c} u_{t}=\omega_{t}-t \omega .
$$

It follows from [WY16, Proposition 9] that

$$
\Delta^{\prime} \log S \geq\left[\frac{t}{n}+\frac{(n+1) \kappa}{2 n}\right] S-1,
$$

where $\Delta^{\prime}$ is the Laplacian of $\omega_{t}$ and $\kappa>0$ is a constant such that $-\kappa$ is the upper bound of the holomorphic sectional curvature $H(\omega)$ of $\omega$. Thus,

$$
S \leq \frac{2 n}{\kappa(n+1)}
$$

On the other hand, applying the maximum principle to $(\mathrm{MA})_{t}$ yields

$$
\max _{X} u_{t} \leq C,
$$

where $C>0$ is a generic constant independent of $t$. It follows from the same process in [WY16] that

$$
\left\|u_{t}\right\|_{C^{k+2, \alpha}} \leq C .
$$

This shows the closedness of $I$. In particular, $0 \in I$ with corresponding $u_{0} \in$ $C^{\infty}(X)$. This gives us the desired Kähler-Einstein metric $d d^{c} \log \omega^{n}+d d^{c} u_{0}$.

\section{Quasi-negative holomorphic sectional curvature}

In this section we seek to extend Theorem 1 to the case $(X, \omega)$ has quasinegative holomorphic sectional curvature, i.e., the holomorphic sectional curvature of $\omega$ is less than or equal to zero everywhere on $X$ and is strictly negative at one point of $X$. This curvature condition is considered in our earlier work joint with P. M. Wong [WWY12]. We can prove the following result (compare $[\mathrm{DT}])$ : 
Theorem 2. Let $(X, \omega)$ be a compact Kähler manifold with quasi-negative holomorphic sectional curvature. Then

$$
\int_{X} c_{1}\left(K_{X}\right)^{n}>0
$$

The proof uses the following two lemmas.

Lemma 3. Let $\Theta$ be a $(1,1)$ form on a compact Kähler manifold $(X, \omega)$ which admits a smooth potential on every coordinate chart $U$, i.e., $\Theta=$ $d d^{c} h_{U}$ for some $h_{U} \in C^{\infty}(U)$. Then for any smooth function $u$ on $X$ satisfying $\Theta+d d^{c} u \geq 0$ on $X$,

$$
\int_{X} e^{-\beta\left(u-\max _{X} u\right)} \omega^{n}<C,
$$

where $\beta$ and $C$ are positive constants depending only on $\Theta$ and $\omega$.

The proof of Lemma 3 is essentially a globalization of the local estimate in [Hör90, p. 97, Theorem 4.4.5] via the Green's formula on a compact manifold, which becomes standard by now. In fact, Lemma 3 has been known to the second author since the late 1970s.

The next lemma can be viewed as a special case of the second author with Cheng [CY75, p. 335, Theorem 1].

Lemma 4. Let $v$ be a negative $C^{2}$ function on a compact Kähler manifold $(X, \omega)$. Suppose the Laplacian $\Delta_{\omega} v \geq-\varphi$ for some continuous function $\varphi$ on $X$. Then,

$$
\int_{X}|\nabla \log (-v)|^{2} \omega^{n} \leq \frac{1}{\min _{X}(-v)} \int_{X}|\varphi| \omega^{n} .
$$

Proof of Lemma 4. Note that

$$
\Delta \log (-v)=\frac{\Delta v}{v}-|\nabla \log (-v)|^{2}
$$

where we abbreviate $\Delta=\Delta_{\omega}$. Integrating both sides against $\omega^{n}$ over $X$ yields

$$
\int_{X}|\nabla \log (-v)|^{2}=\int_{X} \frac{-\Delta v}{-v} \leq \int_{X} \frac{|\varphi|}{\min (-v)},
$$

which is the desired estimate. 
Corollary 5. Let $\Theta$ and u satisfy the condition of Lemma 3. That is, let $\Theta$ be a $(1,1)$ form on a compact Kähler manifold $(X, \omega)$ admitting a smooth potential on every coordinate chart $U$, and let $u \in C^{\infty}(X)$ satisfy $\Theta+d d^{c} u \geq 0$ on $X$. Then $v \equiv u-\max _{X} u-1$ satisfies

$$
\int_{X}|\log (-v)|^{2} \omega^{n}+\int_{X}|\nabla \log (-v)|^{2} \omega^{n} \leq C
$$

where $C>0$ is a constant depending only on $\Theta$ and $\omega$. Consequently, for any sequence $\left\{u_{l}\right\}_{l=1}^{\infty}$ of smooth functions on $X$ satisfying $\Theta+d d^{c} u_{l} \geq 0$, the sequence

$$
\left\{\log \left(1+\max _{X} u_{l}-u_{l}\right)\right\}_{l=1}^{\infty}
$$

is relatively compact in $L^{2}(X)$.

Proof of Corollary 5. By the hypothesis $\Theta+d d^{c} u \geq 0$ we have

$$
\Delta_{\omega} u \geq-\operatorname{tr}_{\omega} \Theta .
$$

Applying Lemma 4 to $v=u-\max _{X} u-1$ yields

$$
\int_{X}|\nabla \log (-v)|^{2} \omega^{n} \leq \int_{X}\left|\operatorname{tr}_{\omega} \Theta\right| \omega^{n},
$$

since $\min _{X}(-v)=1$. To bound the $L^{2}$-norm of $\log (-v)$, recall Lemma 3 that

$$
\int_{X} e^{-\beta\left(u-\max _{X} u-1\right)} \omega^{n} \leq C \int_{X} e^{\beta} \omega^{n}
$$

where the constants $C>0$ and $\beta>0$ depend only on $\Theta$ and $\omega$. Observe that

$$
t \geq \log t, \quad \text { for all } t \geq 1 \text {. }
$$

Then,

Hence,

$$
e^{t} \geq \frac{t^{N}}{N !} \geq \frac{(\log t)^{N}}{N !} \quad \text { for all } N \geq 0, t \geq 1
$$

$$
e^{\beta(-v)} \geq \frac{[\log (-v)]^{N \beta}}{(N !)^{\beta}} .
$$

Choose a large integer $N$ such that $N \beta \geq 2$. Since $\beta$ depends only on $\Theta$ and $\omega$, so is $N$. Applying Hölder's inequality, if needed, yields

$$
\int_{X}[\log (-v)]^{2} \omega^{n} \leq C_{1},
$$


where $C_{1}>0$ is a constant depending only on $\Theta$ and $\omega$. Therefore, we have established inequality (4), which implies the second statement, in view of the standard Rellich Lemma (see [Heb99, p. 37, Theorem 2.9] for example).

Let us now proceed to show Theorem 2.

Proof of Theorem 2. We shall use the continuity method, as in the proof of Theorem 1. Let $u_{t} \in C^{\infty}(X), t>0$, be the solution of

$$
\omega_{t}^{n}=\left(t \omega+d d^{c} \log \omega^{n}+d d^{c} u_{t}\right)^{n}=e^{u_{t}} \omega^{n}
$$

with $\omega_{t} \equiv t \omega+d d^{c} \log \omega^{n}+d d^{c} u_{t}>0$ on $X$. Let $I$ be the interval defined by (1) in the previous proof. Then, $I$ contains a large number $t_{1}$ and $I$ is open, by the same argument in the proof of Theorem 1.

We claim that $I$ contains every $t$ in the interval $\left(0, t_{1}\right]$ provided that the holomorphic sectional curvature $H(\omega) \leq 0$ (which, in particular, implies that $K_{X}$ is nef if $H(\omega) \leq 0$; compare [TY]). Fix an arbitrary $t_{2} \in\left(0, t_{1}\right)$. For any $t_{2} \leq t \leq t_{1}$, by $(2)$ we have

$$
\Delta^{\prime} \log S \geq\left[\frac{t}{n}+\frac{(n+1) \kappa}{2 n}\right] S-1 \geq \frac{t}{n} S-1,
$$

since $H(\omega) \leq-\kappa$ with $\kappa \geq 0$ on $X$. By the standard maximum principle,

$$
\max _{X} S \leq \frac{n}{t} \leq \frac{n}{t_{2}}, \quad \text { for all } t \geq t_{2}
$$

On the other hand, applying the maximum principle to the Monge-Ampère equation yields

$$
\max _{X} u_{t} \leq C \quad \text { for all } t>0
$$

Here and below, we denote by $C>0$ a constant depending only on $n$ and $\omega$, unless otherwise indicated. Proceeding as [WY16] yields

$$
C t_{2} \omega \leq \omega_{t} \leq \frac{C}{t_{2}^{n-1}} \omega
$$

and

$$
\inf _{X} u_{t} \geq C \log t_{2}
$$

This implies the estimate for $u_{t}$ up to the second order. The estimate constants may depend on $t_{2}$, which is fixed. One can then apply, either the local 
Hölder estimate of the second order, or the third order estimate, to obtain a bound for the $C^{2, \alpha}(X)$-norm of $u_{t}$; and hence a bound for $C^{k, \alpha}(X)$-norm of $u_{t}$. The bound may depend on $t_{2}$. This show the closedness of $I$ up to the subinterval $\left[t_{2}, t_{1}\right]$. In particular, $t_{2} \in I$. Since $t_{2}$ is arbitrary, we have proven the claim.

Using the claim, to prove (3) it is equivalent to show

$$
\limsup _{t \rightarrow 0} \int_{X} \omega_{t}^{n}>0
$$

since

$$
\int_{X} \omega_{t}^{n}=\int_{X} c_{1}\left(K_{X}\right)^{n}+n t \int_{X} c_{1}\left(K_{X}\right)^{n-1} \wedge \omega+O\left(t^{2}\right)
$$

as $t \rightarrow 0$. Using inequality (2) again we have

$$
\Delta^{\prime} \log S \geq \frac{(n+1) \kappa}{2 n} S-1 \geq \frac{(n+1) \kappa}{2} \exp \left(-\frac{\max _{X} u_{t}}{n}\right)-1,
$$

where we apply the Netwon-Maclaurin's inequality

$$
S=\frac{\sigma_{n-1}}{\sigma_{n}} \geq n \sigma_{n}^{-1 / n}=n e^{-u_{t} / n}, \quad t>0 .
$$

Integrating against $\omega_{t}^{n}$ over $X$ yields

$$
\begin{aligned}
\exp \left(-\frac{\max _{X} u_{t}}{n}\right) & \leq \frac{\int_{X} \omega_{t}^{n}}{\frac{n+1}{2} \int_{X} \kappa \omega_{t}^{n}} \\
& =\frac{\int_{X} \exp \left(u_{t}-\max _{X} u_{t}-1\right) \omega^{n}}{\frac{n+1}{2} \int_{X} \kappa \exp \left(u_{t}-\max _{X} u_{t}-1\right) \omega^{n}} .
\end{aligned}
$$

Since $t_{1} \omega+d d^{c} \log \omega^{n}+d d^{c} u_{t} \geq \omega_{t}>0$ for each $0<t \leq t_{1}$, applying Corollary 5 with $\Theta=t_{1} \omega+d d^{c} \log \omega^{n}$ yields that the set

$$
\left\{\log \left(1+\max _{X} u_{t}-u_{t}\right) ; 0<t \leq t_{1}\right\}
$$

is relatively compact in $L^{2}(X)$. Then, a sequence $\left\{\log \left(1+\max _{X} u_{t_{l}}-u_{t_{l}}\right)\right\}_{l=1}^{\infty}$ converges in $L^{2}(X)$ to a function $w \in L^{2}(X)$. The standard $L^{p}$ theory (for any $1 \leq p \leq+\infty$; see [AF03, p. 30, Corollary 2.7] for example) implies that a subsequence, still denoted by $\left\{\log \left(1+\max _{X} u_{t_{l}}-u_{t_{l}}\right)\right\}$, converges to $w$ 
almost everywhere on $X$. It follows that

$$
\exp \left(u_{t_{l}}-\max _{X} u_{t_{l}}-1\right) \longrightarrow \exp \left(-e^{w}\right)
$$

almost everywhere on $X$, as $l \rightarrow+\infty$. Since $\exp \left(u_{t_{l}}-\max _{X} u_{t_{l}}-1\right) \leq 1$ for each $l$, it follows from the Lebesgue dominated convergence theorem that $\exp \left(-e^{w}\right) \in L^{1}(X)$ and

$$
\int_{X} \exp \left(u_{t_{l}}-\max _{X} u_{t_{l}}-1\right) \omega^{n} \longrightarrow \int_{X} \exp \left(-e^{w}\right) \omega^{n}>0 .
$$

Moreover, since $\kappa \geq 0$ on $X$ and $\kappa>0$ in an open subset of $X$, we have

$$
\int_{X} \kappa \exp \left(u_{t_{l}}-\max _{X} u_{t_{l}}-1\right) \omega^{n} \longrightarrow \int_{X} \kappa \exp \left(-e^{w}\right) \omega^{n}>0
$$

Plugging these back to (7) yields

$$
\lim _{l \rightarrow+\infty}\left(\max _{X} u_{t_{l}}\right) \geq-C
$$

where the constant $C>0$ depends only on $n$ and $\omega$.

From (8) we have two slightly different ways to conclude (6). For the first way, note that combining (8) and the previous upper bound (5) we know the constant sequence $\left\{\max _{X} u_{t_{l}}\right\}_{l=1}^{\infty}$ is bounded. Thus, by further passing to a subsequence we can assume $u_{t_{l}}$ converges to $\left(-e^{w}+c\right)$ almost everywhere on $X$, where $c$ is a constant. By the upper bound (5) we can apply the Lebesgue dominated convergence theorem to conclude

$$
\int_{X} \omega_{t_{l}}^{n}=\int_{X} e^{u_{t_{l}}} \omega^{n} \longrightarrow \int_{X} \exp \left(-e^{w}+c\right) \omega^{n}>0
$$

as $l \rightarrow+\infty$. This proves the desired (6).

A second way to see (6) is to plug (8) back to the integral inequality in Lemma 3 (with $\Theta=t_{1} \omega+d d^{c} \log \omega^{n}$ ). This gives

$$
\int_{X} e^{-\beta u_{t_{l}}} \omega^{n} \leq C \quad \text { for all } l \geq 1
$$


where $\beta>0$ is a constant depending only on $\omega$. It follows that

$$
\begin{aligned}
\int_{X} \omega^{n} & =\int_{X} e^{-\frac{\beta}{\beta+1} u_{t_{l}}} e^{\frac{\beta}{\beta+1} u_{t_{l}}} \omega^{n} \\
& \leq\left(\int_{X} e^{-\beta u_{t_{l}}}\right)^{\frac{1}{\beta+1}}\left(\int_{X} e^{u_{t_{l}}} \omega^{n}\right)^{\frac{\beta}{\beta+1}} \quad \text { (by Hölder's inequality) } \\
& \leq C^{\frac{1}{\beta+1}}\left(\int_{X} \omega_{t_{l}}^{n}\right)^{\frac{\beta}{\beta+1}} .
\end{aligned}
$$

Hence,

$$
\int_{X} \omega_{t_{l}}^{n} \geq C^{-1 / \beta}\left(\int_{X} \omega^{n}\right)^{\frac{\beta+1}{\beta}}>0, \quad \text { for all } l \geq 1 .
$$

This again confirms (6). The proof of Theorem 2 is therefore completed.

Corollary 6. Let $(X, \omega)$ be a compact Kähler manifold with quasi-negative holomorphic sectional curvature. Then, the canonical bundle $K_{X}$ is ample.

Proof. It is well-known that $X$ contains no rational curve if the holomorphic curvature is nonpositive (see [Roy80, p. 555, Corollary 2] for example). If $X$ is projective, then Theorem 2 implies $K_{X}$ is ample, in view of [WY16, Lemma 5]. When $X$ is Kähler, Theorem 2 implies $K_{X}$ is big, by [MM07, p. 114, Corollary 2.3.38, or Corollary 2.4.2], as we have shown $K_{X}$ is nef in the proof. Thus, $X$ is Moishezon, as a consequence of the standard fact (see [MM07, p. 88, Theorem 2.2.15] for example). Then, a theorem of Moishezon tells us that $X$ is projective (see [MM07, p. 95, Theorem 2.2.26] for example); hence, $K_{X}$ is ample by reducing to the projective case.

\section{Appendix A. On the relative compactness}

After this paper uploaded in arXiv, we realize that Corollary 5 (and also Lemma 4) can be replaced by Lemma 7 below, for which we only need the case $p=1$. The proof of Lemma 7 does not need Lemma 3; in fact, Lemma 7 only requires a lower bound for $\Delta_{\omega} v$, which is much weaker than the semipositivity of $\Theta+d d^{c} v$ in Corollary 5 . As a consequence, Lemma 3 can be bypassed in the proof of Theorem 2 . 
Lemma 7. Let $(X, \omega)$ be an n-dimensional compact Kähler manifold, and let $v$ be a negative $C^{2}$ function satisfying the Laplacian inequality

$$
\Delta_{\omega} v \geq-C_{0}
$$

for some constant $C_{0}>0$ on $X$. Then, for any $1 \leq q<n /(n-1)$,

$$
\begin{aligned}
& \left(\int_{X}|\log (-v)|^{q} \omega^{n}\right)^{1 / q}+\int_{X}|\nabla \log (-v)|^{2} \omega^{n} \\
\leq & C\left[\frac{1}{\min _{X}(-v)}+\min _{X} \log (-v)\right]
\end{aligned}
$$

where $C>0$ is a constant depending only on $n, \omega, q$ and $C_{0}$. Consequently, if a sequence $\left\{v_{l}\right\}_{l=1}^{\infty}$ of $C^{2}$ functions $v_{l}$ satisfies $\Delta_{\omega} v_{l} \geq-C_{0}$, and $-C_{2} \leq$ $\max _{X} v_{l} \leq-C_{1}$ for some positive constants $C_{0}, C_{1}, C_{2}$ independent of $l$, then the sequence $\left\{\log \left(-v_{l}\right)\right\}_{l=0}^{\infty}$ is relatively compact in $L^{p}(X)$ for each $1 \leq$ $p<2 n /(2 n-3)$.

Proof. As in the proof of Lemma 4 we integrate

$$
\Delta \log (-v)=\frac{-\Delta v}{-v}-|\nabla \log (-v)|^{2}
$$

to obtain

$$
\int_{X}|\nabla \log (-v)|^{2} \omega^{n} \leq \frac{C_{0}}{\min _{X}(-v)} \int_{X} \omega^{n}
$$

On the other hand, it follows from (A.1) that

$$
\Delta \log (-v) \leq \frac{C_{0}}{\min _{X}(-v)} \quad \text { on } X .
$$

Applying the standard weak Harnack inequality (see for example [GT01, p. 194, Theorem 8.18]) yields that, for any $1 \leq q<(2 n) /(2 n-2)=n /(n-$ 1),

$$
\left(\int_{X}|\log (-v)|^{q} \omega^{n}\right)^{1 / q} \leq C\left[\min _{X} \log (-v)+\frac{1}{\min _{X}(-v)}\right]
$$

where $C$ depends only on $n, q, C_{0}$, and $\omega$. Combining these two estimates yields the desired inequality. In particular, $\log (-v)$ belongs to the Sobolev space $W^{1, q}(X)$ for any $1 \leq q<n /(n-1)$. Then, the second statement follows immediately from the standard Rellich-Kondrakov theorem (see [Heb99, p. 37, Theorem 2.9] for example), where $p<2 n q_{0} /\left(2 n-q_{0}\right)$ with $q_{0}=n /(n-$ 1) gives $p<2 n /(2 n-3)$. 


\section{References}

[AF03] Robert A. Adams and John J. F. Fournier, Sobolev spaces, volume 140 of Pure and Applied Mathematics (Amsterdam), Elsevier/Academic Press, Amsterdam, second edition, 2003.

[CY75] Shiu-Yuen Cheng and Shing-Tung Yau, Differential equations on Riemannian manifolds and their geometric applications, Comm. Pure Appl. Math. 28(3):333-354, 1975.

[DT] Simone Diverio and Stefano Trapani, Quasi-negative holomorphic sectional curvature and positivity of the canonical bundle, arXiv: 1606.01381.

[GT01] David Gilbarg and Neil S. Trudinger, Elliptic partial differential equations of second order, Classics in Mathematics, SpringerVerlag, Berlin, 2001. Reprint of the 1998 edition.

[Heb99] Emmanuel Hebey, Nonlinear analysis on manifolds: Sobolev spaces and inequalities, volume 5 of Courant Lecture Notes in Mathematics, New York University, Courant Institute of Mathematical Sciences, New York; American Mathematical Society, Providence, RI, 1999.

[Hör90] Lars Hörmander, An introduction to complex analysis in several variables, volume 7 of North-Holland Mathematical Library, North-Holland Publishing Co., Amsterdam, third edition, 1990.

[MM07] Xiaonan Ma and George Marinescu, Holomorphic Morse inequalities and Bergman kernels, volume 254 of Progress in Mathematics, Birkhäuser Verlag, Basel, 2007.

[Roy80] H. L. Royden, The Ahlfors-Schwarz lemma in several complex variables, Comment. Math. Helv. 55(4):547-558, 1980.

[TY] Valentino Tosatti and Xiaokui Yang, An extension of a theorem of $W u$-Yau, arXiv: 1506.01145, to appear in Journal Differential Geometry.

[WWY12] Pit-Mann Wong, Damin Wu, and Shing-Tung Yau, Picard number, holomorphic sectional curvature, and ampleness, Proc. Amer. Math. Soc. 140(2):621-626, 2012.

[WY16] Damin Wu and Shing-Tung Yau, Negative holomorphic curvature and positive canonical bundle, Invent. Math. 204(2):595-604, 2016. 
[Yau78] Shing-Tung Yau, On the Ricci curvature of a compact Kähler manifold and the complex Monge-Ampère equation. I Comm. Pure Appl. Math. 31(3):339-411, 1978.

Department of Mathematics, University of Connecticut 341 Mansfield Road, Storrs, CT 06269-1009, USA E-mail address: damin.wu@uconn.edu

Department of Mathematics, Harvard University One Oxford Street, Cambridge MA 02138, USA

E-mail address: yau@math.harvard.edu

Received OCtober 10, 2016 